\title{
Effects of Topical Tamoxifen on Wound Healing of Burned Skin in Rats
}

\author{
Shaban Mehrvarz ${ }^{1}$, Ali Ebrahimi ${ }^{1}$, Hedayat Sahraei ${ }^{2}$, Mohammad Hasan Bagheri ${ }^{1}$, Sima Fazili ${ }^{3}$, \\ Shahram Manoochehry ${ }^{1}$, Hamid Reza Rasouli ${ }^{1}$ \\ ${ }^{1}$ Trauma Research Center, Baqiyatallah University of Medical Sciences, Tehran; ${ }^{2}$ Department of Physiology and Biophysics, and Behavioral \\ Sciences Research Center, Baqiyatallah University of Medical Sciences, Tehran; ${ }^{3}$ Department of Pathology, Baqiyatallah University of Medical \\ Sciences, Tehran, Iran
}

Background This study aimed to assess the effects of the topical application of tamoxifen on wound healing of burned skin in Wistar rats by evaluating 3 healing characteristics: fibrotic tissue thickness (FT), scar surface area (SSA), and angiogenesis in the healed scar tissue.

Methods Eighteen male Wistar rats were used in this study. A third-degree burn wound was made on the shaved animals' back, measuring $2 \times 2 \times 2 \mathrm{~cm}$. In the first group, a $2 \%$ tamoxifen ointment was applied to the wound twice daily for 8 weeks. The second group received a placebo ointment during the same period. The third group did not receive any treatment and served as the control group.

Results The median (interquartile range $=[01,03]) \mathrm{FT}$ was $1.35(1.15,1.62) \mathrm{mm}, 1.00(0.95$, 1.02) $\mathrm{mm}$, and $1.25(0.8,1.5) \mathrm{mm}$ in the control, tamoxifen, and placebo groups, respectively $(P=0.069)$. However, the $F T$ in the tamoxifen group was less than in the placebo and control groups. The median angiogenesis was $3.5(3.00,6.25), 8.00(6.75,9.25)$, and $7.00(5.50,8.25)$ vessels per high-power field for the control, tamoxifen, and placebo groups, respectively $(P=0.067)$. However, the median angiogenesis was higher in the tamoxifen group than in the control group. No significant difference was observed in the mean SSA between the tamoxifen group and the control group $(\mathrm{P}=0.990)$.

Conclusions Local application of tamoxifen increased angiogenesis and decreased the FI, with no change in the SSA in burned skin areas. These effects are expected to expedite the wound healing process, reducing contracture and preventing hypertrophic scar and keloid formation.

Keywords Tamoxifen / Wound healing / Burns / Keloid / Rats

\author{
Correspondence: \\ Shahram Manoochehry \\ Trauma Research Center, Baqiyatallah \\ University of Medical Sciences, \\ 1435917541, Mollasadra Street, \\ Sheikh Bahaee Street, Tehran, Iran \\ Tel: +98-2188053766 \\ Fax: +98-2188037561 \\ E-mail: \\ shahram.manoochehry@yahoo.com
}

We thank Dr. Ali Zamani, the Quality Assurance Manager of the Pars Darou Pharmaceutical Company for preparing the drug, and Dr. Mohammad Hosien Kalantar Motamedi for his kind assistance in editing this paper.

No potential conflict of interest relevant to this article was reported.

\section{INTRODUCTION}

Excessive scar formation after deep burns is inevitable. In the healing process, hypertrophic scars, keloids, and contracture can occur, which in turn cause emotional, social, and functional problems for the rest of the victim's life.

The pathophysiology of the formation of hypertrophic scars, keloids, and contracture is not yet completely understood; for 
this reason, both surgical and non-surgical treatment modalities have yielded disappointing results.

Considering the high costs of excessive scar treatment despite various therapeutic strategies, controversies still exist regarding the best treatment. Therefore, it has been suggested that preventing excessive scar formation may be more effective [1].

Prevention of abnormal scarring after skin burns is a challenging problem, and several therapies exist for the reduction of hypertrophic scar and keloid formation. Tamoxifen is a nonsteroidal antagonist of estradiol used extensively in the prevention and treatment of breast cancer. This drug reducing the production of collagen by decreasing transforming growth factor (TGF- $\beta 2$ ), and can prevent fibroblast formation in wound healing. It is also implicated in the reduction of hypertrophic scar and keloid formation. Previous studies have shown the effects of tamoxifen on inhibiting fibroblasts in Dupuytren contracture affecting the palmar fascia [2], keloids [3] and retroperitoneal fibrosis [4]. In an in vitro study, it was shown that exposure to tamoxifen in human dermal cells could decrease cell growth factors (basic fibroblast growth factor and vascular endothelial growth factor), and it was suggested that the clinical use of tamoxifen may decrease scar formation [5]. A randomized controlled trial of 300 patients with a history of hypertrophic scarring showed that oral tamoxifen could significantly reduce hypertrophic scars after surgical incisions [6].

Tamoxifen prevents myofibroblast differentiation and may have a therapeutic role in chronic inflammatory conditions or cancer [7]. Prevention of keloid and hypertrophic scar formation is still a challenging field, and several different therapeutic modalities have been reviewed by Berman et al. [8] .

\section{Fig. 1. An anaesthetized rat with a shaved back}

Rats were anaesthetized and the dorsal hair was shaved in a $3 \times 3 \mathrm{~cm}$ area.

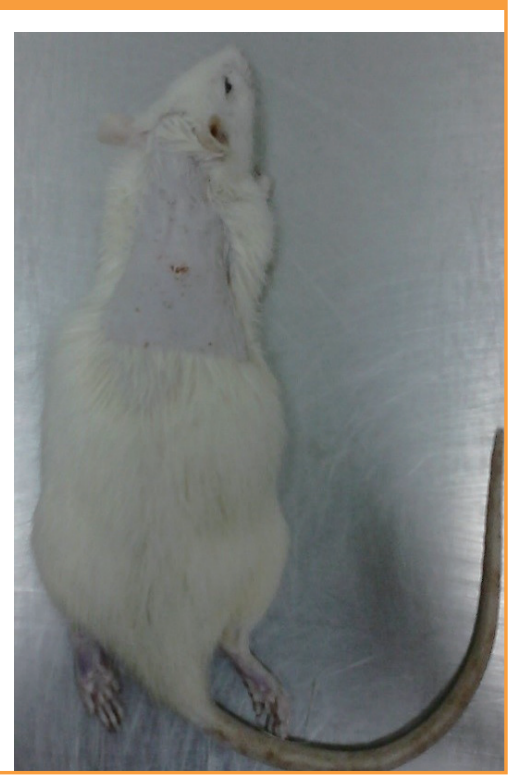

This study aimed to assess the effects of the topical application of tamoxifen on wound healing of burned skin in Wistar rats by evaluating 3 healing characteristics: fibrotic tissue thickness (FTT), scar surface area (SSA), and angiogenesis in healed scar tissue.

\section{METHODS}

Eighteen male Wistar rats with a mean weight of 250 to $300 \mathrm{~g}$ were used in this study. The rats were obtained from our hospital animal experimental laboratory, and the study was conducted in the same laboratory. The rats were randomly divided into 3 groups, all with an equal number of animals. All animals were anesthetized via intraperitoneal injection of ketamine $(60 \mathrm{mg} /$ $\mathrm{kg}$ ), and a 3- $\times 3-\mathrm{cm}$ area of dorsal hair was shaved (Fig. 1). A third-degree burn wound was made with a $2-\times 2-\times 2-\mathrm{cm}$ stainless steel cube (Fig. 2) that was placed in $96^{\circ} \mathrm{C}$ water for $3 \mathrm{~min}$ utes and then was placed on the shaved area for 15 seconds (Fig. 3). All animals were kept under standard laboratory conditions and were provided with identical food and water during the study period. After recovery, rats were individually housed in separate cages. The cages were cleaned daily and kept free from infectious agents. In 2 of the groups, a $2 \%$ tamoxifen ointment or a placebo ointment was applied to the wound twice daily for 8 weeks. Each of the animals was kept in a separate cage to prevent licking of the applied ointment by other mice, so we did not use any cover or dressing over the wounds after applying the ointment.

In the first group, a $2 \%$ tamoxifen ointment was applied to the wound twice daily for 8 weeks. The second group received a

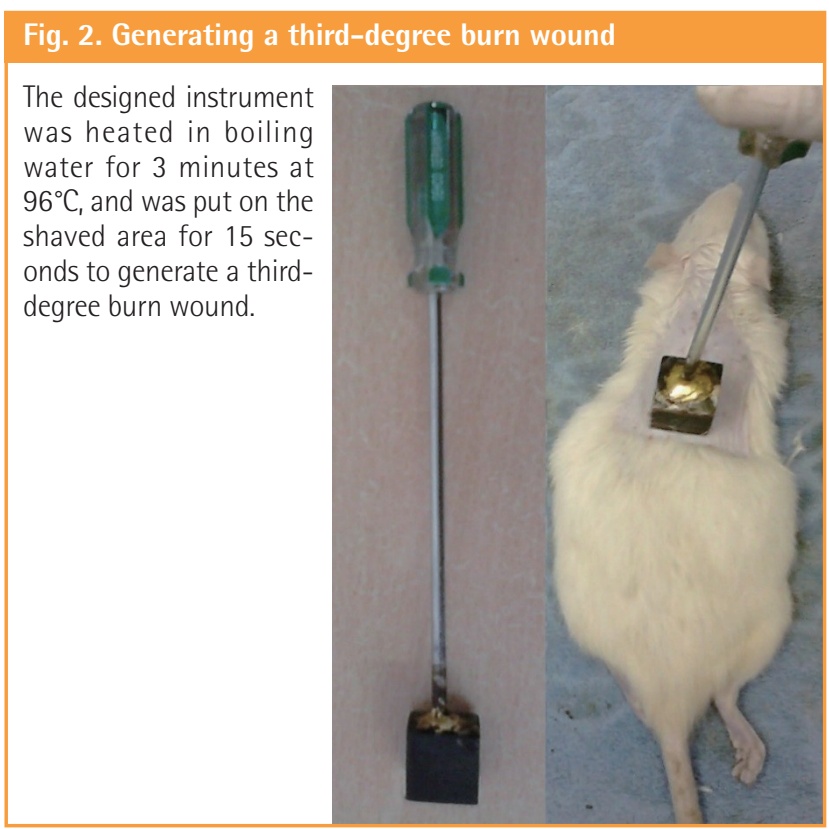


placebo ointment during the same period. The third group did not receive any treatment and served as the control group. Tamoxifen and the placebo ointment were provided in similar tubes. Both tamoxifen and the placebo ointment were purchased from the Iran Hormone Pharmaceutical Company, Tehran, Iran. The $2 \%$ tamoxifen and placebo ointments were prepared in 2 differently colored containers without any labels. This drug made only for our experiment by the pharmaceutical company Pars Darou. The ingredients of the placebo ointment were a combination of glyceryl monostearate, liquid paraffin, petrolatum, propylene glycol, cetyl alcohol, and isopropyl myristate.

The ointment was the vehicle only, with no active ingredient. Throughout the entire period of this study, none of the investigators knew which ointment was tamoxifen, and each animal had a private code number, which was used as an identification

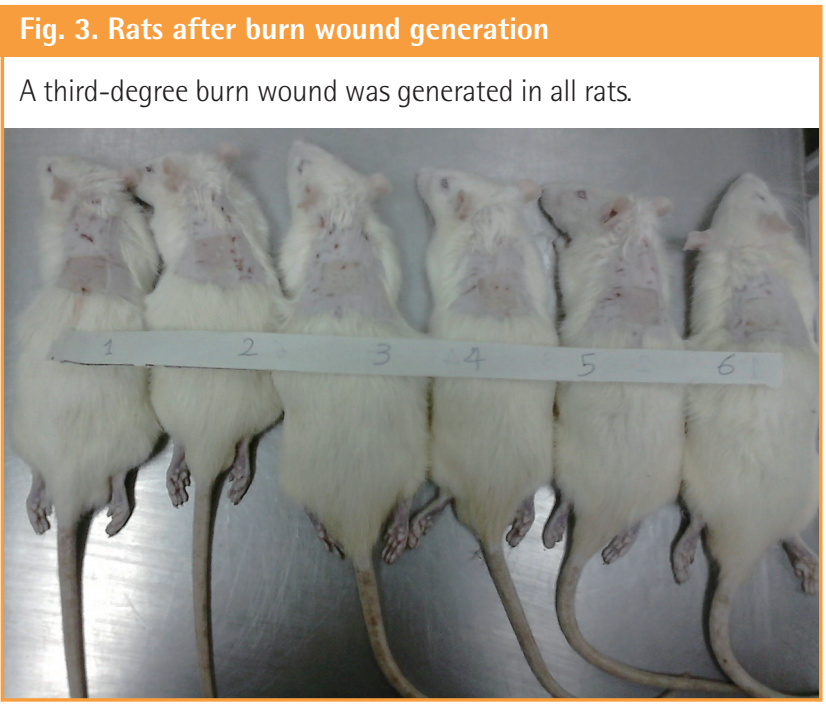

code for pathology.

The protocol of the study was approved by the Animal Medical Ethics Committee of Baqiyatallah University of Medical Sciences (Ref. letter no. s/340/3/5694). In order to collect specimens, the animals were euthanized at 8 weeks after the burn injury was made, under a high concentration of chloroform (Fig. 4).

\section{Histopathological assessment}

The wounds were photographed on the first and last day of the study. The SSA was calculated in square millimeters. For pathologic evaluation, excisional biopsies from the thickest area of the scar were performed, and the amount of fibrosis and angiogenesis were assessed via a microscopic examination. Hematoxylin and eosin staining was used to evaluate angiogenesis (vessels per high-power field, HPF) (Fig. 5), and Masson trichrome staining

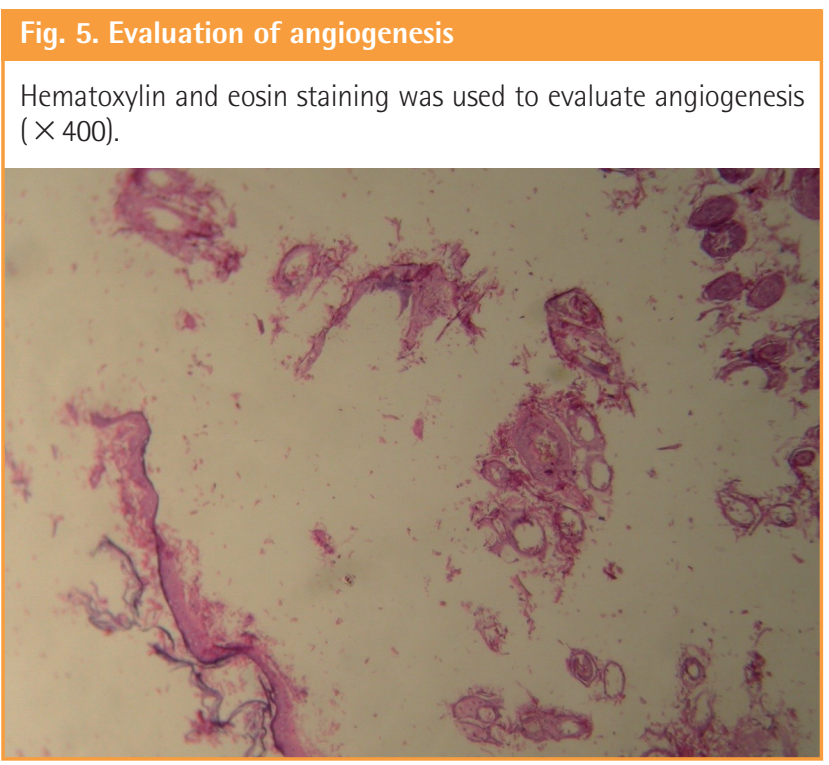

Fig. 4. Wounds on the last day of the experiment

Gross view of wounds on the last day of the experiment. (A) A rat from the control group. (B) A rat from the placebo group. (C) A rat from the tamoxifen group. The arrows point to the wound scar sites.

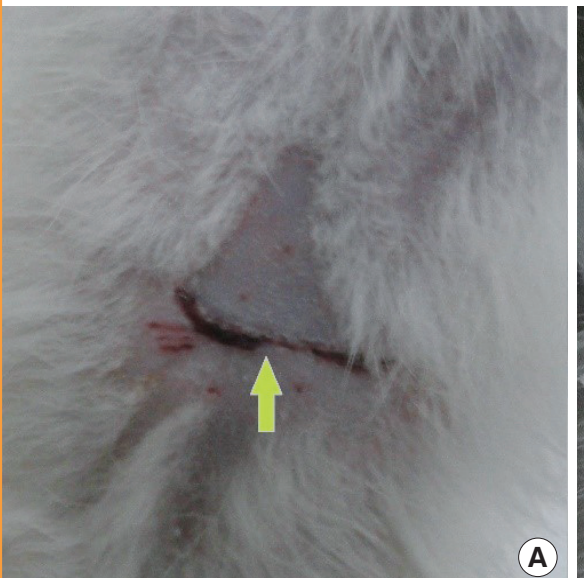

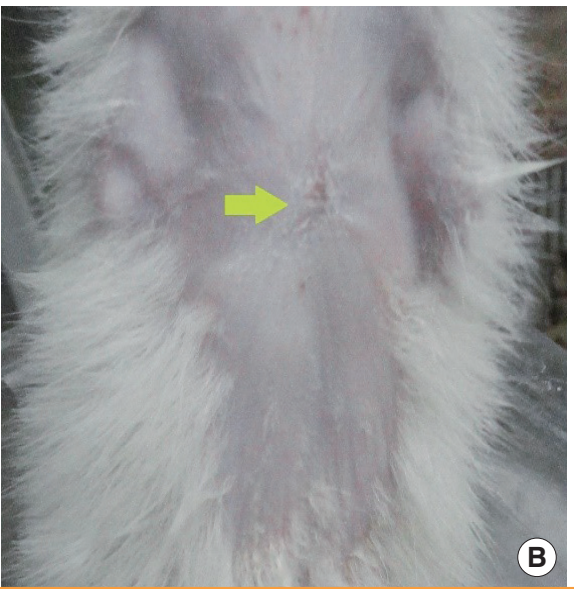

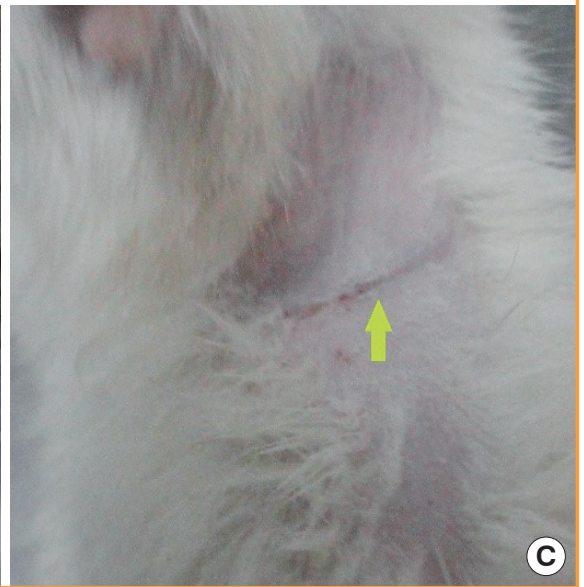


was used to measure the FTT in millimeters (Fig. 6). All measurements were conducted by 2 independent blinded pathologists. All participants in the project, including the laboratory staff, wound assessors, and the data analyzer were blinded to the group allocation and treatments.

\section{Data analysis}

Data analysis was performed using SPSS ver. 24.0 (IBM Corp., Armonk, NY, USA). Descriptive statistics such as median and the interquartile range (IQR; Q1, Q3) were used to present the results. The Kruskal-Wallis test was performed to analyze the differences among the 3 groups. P-values of $<0.05$ were considered to indicate statistical significance. Pairwise comparisons between the groups were performed using the Dunn method.

\section{RESULTS}

A summary of the data analysis results and comparisons across groups is presented in Table 1 . At the end of the study, the median $(\mathrm{IQR}=[\mathrm{Q} 1, \mathrm{Q} 3]) \mathrm{FTT}$ was $1.35(1.15,1.62) \mathrm{mm}, 1.00$

\section{Fig. 6. Measurements of fibrotic tissue thickness}

Masson trichrome staining was used to measure fibrotic tissue thickness $(\times 400)$.

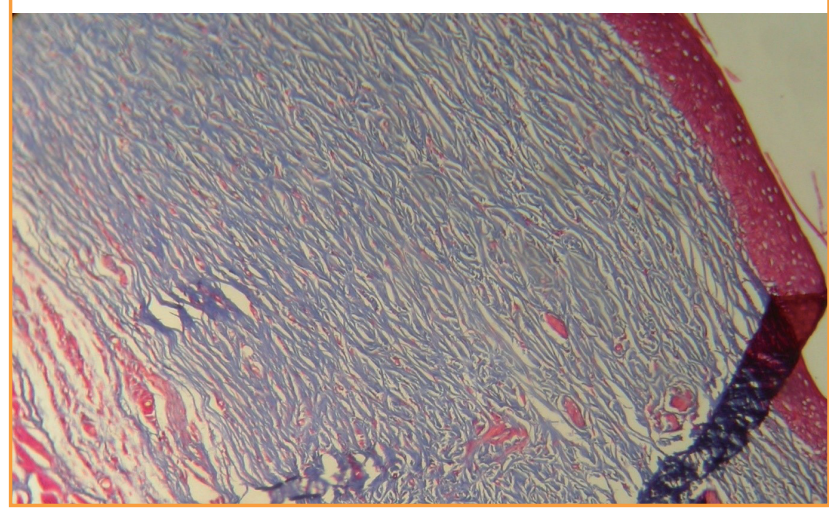

$(0.95,1.02) \mathrm{mm}$, and $1.25(0.8,1.5) \mathrm{mm}$ in the control, tamoxifen, and placebo groups, respectively.

No significant difference was observed in the FTT across the groups $(\mathrm{P}=0.069)$. However, the FTT in the tamoxifen group was less than in the control and placebo groups. The median $(\mathrm{IQR}=[\mathrm{Q} 1, \mathrm{Q} 3])$ angiogenesis was 3.5 (3.00, 6.25), 8.00 (6.75, $9.25)$ and $7.00(5.50,8.25)$ vessels/HPF for the control, tamoxifen, and placebo groups, respectively. The median angiogenesis was higher in the tamoxifen group than in the control group. However, these differences between the groups was not statistically significant $(\mathrm{P}=0.067)$.

The SSA was $89.00(69.00,112.50) \mathrm{mm}^{2}, 77.50$ (52.00, $100.00) \mathrm{mm}^{2}$, and $49.00(39.00,60.00) \mathrm{mm}^{2}$ for the control, tamoxifen, and placebo groups, respectively. However, no significant difference was observed between the tamoxifen group and the control group $(\mathrm{P}=0.990)$. However, the SSA was significantly smaller in the placebo group than in the tamoxifen group $(\mathrm{P}=0.032)$.

\section{DISCUSSION}

This study found that local application of tamoxifen ointment could reduce excessive scar formation and wound contraction in third-degree burned skin. This effect may have been caused by inhibiting fibrin accumulation, facilitating the process of healing, and maintaining and increasing the blood supply to the wound.

The present study showed that tamoxifen reduced the thickness of the fibrotic tissue; however, this was not statistically significant, which might have been due to the limited number of samples. The clinical importance of this issue is that the prevention of hypertrophic scar and keloid formation is the most challenging problem in patients with severe burns.

In the present study, a significant decrease was observed in the final surface area of the scar in the placebo group compared with

Table 1. Comparison of tamoxifen with the control and placebo

\begin{tabular}{|c|c|c|c|}
\hline Items & Fibrotic tissue (thickness, mm) & Angiogenesis (vessels/HPF) & Scar surface area $\left(\mathrm{mm}^{2}\right)$ \\
\hline Tamoxifen & $1.00(0.95,1.02)$ & $8.00(6.75,9.25)$ & $77.50(52.00,100.00)$ \\
\hline Control & $1.35(1.15,1.62)$ & $3.50(3.00,6.25)$ & $89.00(69.00,112.50)$ \\
\hline Placebo & $1.25(0.80,1.50)$ & $7.00(5.50,8.25)$ & $49.00(39.00,60.00)$ \\
\hline P-value & 0.069 & 0.067 & 0.028 \\
\hline \multicolumn{4}{|c|}{ Pairwise comparisons by the Dunn method } \\
\hline$A$ versus $B$ & - -a) $^{\text {a) }}$ & $--^{\text {a) }}$ & 0.990 \\
\hline$A$ versus $C$ & $--^{\text {a) }}$ & - -a) $^{-a}$ & 0.160 \\
\hline$B$ versus $C$ & $--^{\text {a) }}$ & - -a) $^{\text {a) }}$ & 0.032 \\
\hline
\end{tabular}


the tamoxifen group. It appears that wound coverage by the ointment in that group prevented the wound dehydration and facilitated wound re-epithelialization, which consequently increased fibroblast activity and reduced the final scar area. Inhibition of wound contraction is clinically important because it would not only ameliorate problems such as pain, deformity, and limitations in body movements, but also reduce the need for surgical correction [9].

There was no statistically significant reduction in the final surface area of the scars in the tamoxifen group compared with the control group. This was most likely due to the inhibitory effect of tamoxifen on the TGF- $\beta$ activation pathway, which leads to the inhibition of myofibroblast induction; these events ultimately reduce $\alpha$-smooth muscle actin and extracellular matrix proteins (such as collagen and fibronectin) as suppliers of wound contraction power. Thus, wound contraction is reduced. $\mathrm{Hu}$ et al. have also shown that tamoxifen reduces wound contraction by inhibiting the collagen network $[10,11]$.

Tamoxifen pills (Nolvadex) are used to prevent and treat estrogen receptor-positive types of breast cancer. A liquefied form of tamoxifen (Soltamox) is also available. Tamoxifen ointment is used mainly for experimental proposes. Tamoxifen can cause side effects, including weight gain, sexual problems, thromboembolic events, stroke, and neurocognitive problems [12]. These systemic side effects are important, and the long-term use of oral tamoxifen should therefore be avoided.

The mechanisms of the effects of tamoxifen are complex and have not been clearly defined. It binds to estrogen receptors and blocks the proliferative action of estrogen in mammary cells. This action of tamoxifen is induced by the synthesis of the cytokine TGF- $\beta$, which acts as a negative autocrine regulatory molecule $[13]$.

Leventhal et al. [14], in a meta-analysis, reviewed 36 articles published about the outcomes of various types of treatment modalities, and concluded that there was no optimal treatment modality for the management of keloid and hypertrophic scars.

Topical applications for the treatment or prevention of postsurgical skin injuries are noninvasive and more comfortable, and also cost less than other modalities [15].

Mikulec et al. [16] proposed that tamoxifen may improve wound healing in keloids by reducing the expression of TGF- $\beta 1$.

Foo and Tristani-Firouzi [17] reviewed topical modalities for the treatment and prevention of post-surgical hypertrophic scars, argued that there is no universally accepted treatment for the prevention or elimination of hypertrophic scars, and then recommended "massage therapy" for the reduction of post-burn scarring.

Based on the results of this study, it appears that the use of tamoxifen locally on the burned skin of rats caused an increase in angiogenesis, decreased the FTT, and caused no significant change in the final scar size in the burned areas. These factors can expedite the wound healing process by reducing contracture and preventing hypertrophic scar and keloid formation. Moreover, the local use of tamoxifen locally does not carry the risk of systemic side effects of the drug.

\section{REFERENCES}

1. Mustoe TA, Cooter RD, Gold MH, et al. International clinical recommendations on scar management. Plast Reconstr Surg 2002;110:560-71.

2. Kuhn MA, Wang X, Payne WG, et al. Tamoxifen decreases fibroblast function and downregulates TGF (beta2) in dupuytren's affected palmar fascia. J Surg Res 2002;103:14652.

3. Zhao JY, Chai JK, Song HF, et al. Effect of different concentration of tamoxifen ointment on the expression of TGF-beta2 of hypertrophic scar at rabbit ears. Zhonghua Zheng Xing Wai Ke Za Zhi 2011;27:213-7.

4. Owens LV, Cance WG, Huth JF. Retroperitoneal fibrosis treated with tamoxifen. Am Surg 1995;61:842-4.

5. Ruffy MB, Kunnavatana SS, Koch RJ. Effects of tamoxifen on normal human dermal fibroblasts. Arch Facial Plast Surg 2006;8:329-32.

6. Mousavi SR, Raaiszadeh M, Aminseresht M, et al. Evaluating tamoxifen effect in the prevention of hypertrophic scars following surgical incisions. Dermatol Surg 2010;36:665-9.

7. Carthy JM, Sundqvist A, Heldin A, et al. Tamoxifen inhibits TGF- $\beta$-mediated activation of myofibroblasts by blocking non-smad signaling through ERK1/2.J Cell Physio 2015;230: 3084-92.

8. Berman B, Maderal A, Raphael B. Keloids and hypertrophic scars: pathophysiology, classification, and treatment. Dermatol Surg 2017;43 Suppl 1:S3-18.

9. Schouten HJ, Nieuwenhuis MK, van Zuijlen PP. A review on static splinting therapy to prevent burn scar contracture: do clinical and experimental data warrant its clinical application? Burns 2012;38:19-25.

10. Hu D, Hughes MA, Cherry GW. Topical tamoxifen: a potential therapeutic regime in treating excessive dermal scarring? Br J Plast Surg 1998;51:462-9.

11. Hu D, Zhu X, Xu M, et al. The inhibitory effect of tamoxifen on human dermal fibroblast-populated collagen lattices. Zhonghua Zheng Xing Wai Ke Za Zhi 2002;18:160-2.

12. Pemmaraju N, Munsell MF, Hortobagyi GN, et al. Retrospective review of male breast cancer patients: analysis of 
tamoxifen-related side-effects. Ann Oncol 2012;23:1471-4.

13. Sporn MB, Lippman SM. Agents for chemoprevention and their mechanism of action. In: Kufe DW, Pollock RE, Weichselbaum RR, et al., editors. Holland-Frei cancer medicine. 6th ed. Hamilton: BC Decker; 2003.

14. Leventhal D, Furr M, Reiter D. Treatment of keloids and hypertrophic scars: a meta-analysis and review of the literature. Arch Facial Plast Surg 2006;8:362-8.
15. Zurada JM, Kriegel D, Davis IC. Topical treatments for hypertrophic scars. J Am Acad Dermatol 2006;55:1024-31.

16. Mikulec AA, Hanasono MM, Lum J, et al. Effect of tamoxifen on transforming growth factor betal production by keloid and fetal fibroblasts. Arch Facial Plast Surg 2001;3:111-4.

17. Foo CW, Tristani-Firouzi P. Topical modalities for treatment and prevention of postsurgical hypertrophic scars. Facial Plast Surg Clin North Am 2011;19:551-7. 